\title{
Immunotherapy and the Immune Infiltrate in Pediatric Brain Tumors: An Illustration and Review of the Unique Challenges Facing Immunotherapy for Pediatric Oncology
}

\author{
Ashley S Plant ${ }^{1 *}$ and Eugene I Hwang ${ }^{2}$ \\ ${ }^{1}$ Department of Pediatric Neuro-Oncology, Children's Hospital Orange County, USA \\ ${ }^{2}$ Department of Pediatric Neuro-Oncology, Children's National Health System, USA
}

*Corresponding author: Ashley S Plant, MD, Department of Pediatric Neuro-Oncology, Children's Hospital Orange County, 1201 W. La Veta Ave Orange, CA 92868, USA, Tel: 714-509-4348, Fax: 714-509-8699, E-mail: aplant@choc.org

\begin{abstract}
Immunotherapy for pediatric oncology is a robust and prolific area of active research and has changed the face of treatment for some cancers, such as, B cell acute lymphoblastic leukemia (ALL) and neuroblastoma. However, the field faces challenges and hurdles unique to the pediatric population especially in the area of neuro-oncology. Here, clinicians face challenges of using immunotherapy for tumors with some of the lowest mutational burdens in sensitive areas of the brain where surgical intervention is limited and significant immune infiltration is poorly tolerated. Here, we review the current knowledge of the interplay between the immune system and pediatric brain tumors, current clinical trials enrolling patients with pediatric brain tumors, and the challenges unique to this area of research. Early phase clinical trials in pediatric neuro-oncology are plentiful given the rarity of these tumors and efficacy and safety is still to be determined.
\end{abstract}

\section{Keywords}

Immunotherapy, Pediatric brain tumors, Immune infiltrate, Pseudo-progression, Checkpoint blockade, Vaccine, Chimeric antigen receptor (CAR) T cell

\section{Introduction}

Immunotherapy for pediatric oncology is a robust and prolific area of active research and has changed the face of treatment in such cancers as neuroblastoma and the hematologic malignancies. Neuroblastoma is the most common extra-cranial solid tumor in childhood. It originates in the adrenal medulla and paraspinal or periaortic regions where the sympathetic nervous system is present. The
Children's oncology group (COG) conducted a phase III trial (ANBL0931) evaluating the efficacy of ch14.18, a monoclonal antibody to tumor-associated disialoganglioside (GD2) in combination with alternating granulocyte-macrophage colony-stimulating factor (GM-CSF) or interleukin-2 (IL-2) [1]. GD2 is present on the majority of neuroblastoma tumor cells even in heterogeneous tumors and is present on only a limited spectrum of normal cells including peripheral pain fibers [2]. The Fc portion of ch14.18 binds the Fc receptor of monocytes, macrophages, neutrophils, and natural killer cells to stimulate tumor cell lysis via antigen-dependent cell-mediated cytotoxicity (ADCC) and also triggers complement-dependent cytotoxicity (CDC). In a COG study (ANBL0032), patients with high risk neuroblastoma were randomized after completion of chemotherapy, autologous stem cell transplant, and radiation to standard therapy with isotretinoin versus treatment with isotretinoin plus anti-GD2 combined with GM-CSF alternating with IL-2 [3]. The experimental arm was found to be superior with an 2 year event free survival (EFS) of $66 \% \pm$ $5 \%$ vs. $46 \% \pm 5 \%(p=0.01)$ and overall survival (OS) $86 \% \pm$ $4 \%$ vs. $75 \% \pm 5 \%(p=0.02)$. Isotretinoin itself is known to have distinct effects on immune system including changing the levels of natural killer (NK) cells and alterations in the innate immune system $[4,5]$. Immunotherapy improved outcomes for this very high risk group of patients and is now part of the standard of care.

In the area of hematologic malignancies, mature B cell lymphoma patients benefited from the addition 
of rituximab being added to the standard LMB-96 chemotherapy backbone in the COG study ANHL1131 [6]. Rituximab is a genetically engineered chimeric murine/ humanized monoclonal IgG1 kappa antibody directed against the CD20 antigen. In the interim analysis for this study, 1 year EFS was $81.5 \%$ in the control arm compared to $94.2 \%$ in the rituximab arm. Although the primary endpoint of EFS did not cross the pre-specified efficacy boundary, conditional power analysis predicted a high likelihood of a positive study. As a result, the Independent data safety monitoring committee (IDSMC) recommended discontinuation of randomization and that all children with high risk $B$ cell lymphoma received the LMB chemotherapy backbone plus an additional 6 injections of Rituximab.

There has also been success seen in pediatric oncology for adoptive cellular immunotherapy. Chimeric antigen receptor (CAR) T cells combine antigen binding and recognition with $T$ cell activating functions in a single molecule. The chimeric antigen receptor is engineered to have arbitrary specificity on an immune effector cell. As a therapeutic agent, the specificity is typically to a cancer-specific antigen. In recurrent or refractory B cell ALL, CAR T cells directed against CD19+ leukemia cells resulted in an $80 \%$ overall survival at 1 year and were found to persist up to 1 year [7]. The relapse free rate was $75 \%$ at 6 months and $64 \%$ at 12 months, an unprecedented result for a patient group that is heavily pre-treated. Overall survival was $89 \%$ at 6 months and $79 \%$ at 12 months. CD19 CAR T cell therapy is now being considered for incorporation into the next children's oncology group (COG) study for ALL in the setting of positive minimal residual disease after consolidation.

Despite these examples of successes of immunotherapy, there are also unique challenges and hurdles facing utilization of immunotherapy in pediatric oncology. Generally, pediatric tumors may be less antigenic than many adult cancers, carrying much lower mutational loads compared to their adult counterparts [8]. For instance, rhabdoid tumors, seen in the kidneys as well as in the brain, possess only a solitary genetic alteration, a mutation in the SWI/SNF related, matrix associated, actin dependent regulator of chromatin, subfamily $B$, member 1 (SMARCB1) gene. We also know that the pediatric immune system evolves from a neonate to an older child with an initially immature innate and adaptive immune system that acquires memory over time [9]. This may result in variations in the immune response to tumors not only when compared to adults, but even throughout childhood development. There are practical differences in the management of immunotherapy for pediatric tumors as well. For instance, in the area of pediatric brain tumors, two-thirds of the tumors exist in the posterior fossa while only $15 \%$ of adult brain tumors occur in this space. The posterior fossa is an area where pseudoprogression or tumor inflammation related to immunotherapy can result in significant clinical issues, such as hydrocephalus and increased intracranial pressure as well as brainstem dysfunction including apnea or the inability to swallow. However, pediatric patients have decades to accumulate long term sequelae from traditional therapies such as radiation and chemotherapy and may serve to benefit the most from therapies that harness their own immune system into a cytotoxic therapeutic. Here, we will review the case of pediatric brain tumors to illustrate some of the challenges facing immunotherapy for pediatric oncology.

\section{The Immune Infiltrate}

Much is known about the immune infiltrate in adult gliomas. Research has confirmed that there is indeed immune trafficking to the brain and evaluation of adult glioma immune infiltrate has shown a significant difference between the infiltrate of low grade versus high grade glioma [10]. High grade gliomas have evidence of a more robust immune response with more natural killer (NK) cells and a higher T cell infiltrate and CD8: CD4 $\mathrm{T}$ cell ratio but also a more immunosuppressed phenotype with more infiltrating regulatory $T$ cells and macrophages of the $M 2$ subtype. The presence of an increased immune infiltrate, especially CD8 T cells, has also been associated with improved prognosis in adult gliomas [11]. Multiple immune escape mechanisms within the innate and adaptive immune systems have also been identified [12]. In contrast, little is known about the immune infiltrate in pediatric tumors and how this compares to their adult counterparts.

In a previous study, the immune infiltrate of pediatric brain tumors was evaluated using multicolor FACS and gene expression microarray on disaggregated tumor tissue $[13,14]$. In this study, ependymomas and pilocytic astrocytomas showed a larger myeloid infiltrate and a more activated phenotype with HLADR and CD64 positivity. Higher grade lesions, such as, medulloblastoma and glioblastoma had a smaller $T$ cell infiltrate and a larger percentage of memory $T$ cells. From this stu$d y$, the authors concluded that low grade tumors may benefit from vaccine therapies given the presence of a pre-existent immune infiltrate while high grade tumors like medulloblastoma and glioblastoma may benefit from checkpoint blockade to counteract the suppressive immune microenvironment [14].

In another study, FACS analysis was conducted on 22 fresh pediatric brain tumors and corresponding peripheral blood samples. Immunohistochemistry for major immune markers was run on an additional 89 pediatric brain tumor samples [15]. The immune infiltrate was dissimilar to that reported for adult gliomas. Low grade gliomas had a higher $\mathrm{T}$ cell infiltrate than high grade gliomas. This was confirmed with $T$ cell receptor sequencing of the TCR variable beta chain sequencing and clonality analysis. 
Unlike in adult studies, in the pediatric analysis, there was no statistical correlation between immune markers for the CD45 immune subset, CD8 T cells, CD20 B cells, or FoxP3+ regulatory $T$ cells and survival, although this could be due to lower samples numbers compared to adult studies [15]. Mutational load in this series of pediatric brain tumors was low overall but did correlate with the presence of CD8 effector memory T cells, activated memory $T$ cells, activated CD20 B cells, and regulatory $T$ cells as determined by FACS immune subsets. This suggests that low mutational burden could, in fact, be impacting the immunogenicity of these tumors.

Overall, the analysis of the immune infiltrate in pe- diatric brain tumor studies demonstrated disparate results from the adult data, with less impact on prognosis but did have a positive correlation with mutational load. Future studies in pediatric oncology should focus on understanding the interplay between pediatric tumors and the immune system and how this may implicate specific immunotherapies. Extrapolation from adult data should be used with caution.

\section{Clinical Trials in Immunotherapy}

Recent clinical trials for immunotherapy in pediatric brain tumors span the gamut of immunotherapy subtypes (Table 1). In the area of vaccines, the Pacific

Table 1: Provides selected clinical trials currently open in pediatric neuro-oncology that utilize immunotherapy strategies within the categories of Vaccine therapy, Chimeric antigen receptor (CAR) T cell therapy, Checkpoint blockade, and Viral therapies. This table provides Immunotherapy category, Clinicaltrials.gov NCT number, Primary site location, Name of specific therapy, and Eligible disease types.

\begin{tabular}{|c|c|c|c|c|}
\hline \multicolumn{5}{|c|}{ Recent clinical trials: Immunotherapy for pediatric brain tumors } \\
\hline $\begin{array}{l}\text { Type of } \\
\text { immunotherapy }\end{array}$ & $\begin{array}{l}\text { Clinical trials.gov } \\
\text { identifier }\end{array}$ & $\begin{array}{l}\text { Lead institution/ } \\
\text { Consortium }\end{array}$ & Investigational agent & Eligible tumor type \\
\hline \multirow[t]{5}{*}{ Vaccine } & NCT02960230 & $\begin{array}{l}\text { University of california, San } \\
\text { francisco (PNOC) }\end{array}$ & H3.3K27M peptide vaccine & $\begin{array}{l}\text { HLA-A201+ children with } \\
\text { newly diagnosed DIPG } \\
\text { and H3.3K27M positive } \\
\text { glioma }\end{array}$ \\
\hline & NCT01130077 & University of pittsburgh & $\begin{array}{l}\text { Glioma-associated antigen } \\
\text { vaccine (EphA2, IL-13Ra2, } \\
\text { survivin) }\end{array}$ & $\begin{array}{l}\text { HLA-A2+ children with } \\
\text { DIPG or recurrent or } \\
\text { High grade glioma }\end{array}$ \\
\hline & NCT01808820 & $\begin{array}{l}\text { University of miami (HGG } \\
\text { Immuno Group) }\end{array}$ & $\begin{array}{l}\text { Tumor lysate pulsed } \\
\text { dendritic cell vaccine }+ \\
\text { Imiquimod }\end{array}$ & High grade glioma \\
\hline & NCT02722512 & $\begin{array}{l}\text { Lurie children's hospital of } \\
\text { chicago }\end{array}$ & $\begin{array}{l}\text { Heat shock protein peptide } \\
\text { complex-96 (HSPPC-96) } \\
\text { vaccine }\end{array}$ & $\begin{array}{l}\text { Newly diagnosed or } \\
\text { Recurrent high grade } \\
\text { glioma }\end{array}$ \\
\hline & NCT03068832 & Washington university & $\begin{array}{l}\text { Neo-epitope personalized } \\
\text { synthetic long peptide } \\
\text { vaccine }\end{array}$ & $\begin{array}{l}\text { Recurrent malignant } \\
\text { CNS tumors }\end{array}$ \\
\hline \multirow[t]{2}{*}{ CAR T cell } & NCT03500991 & Seattle children's hospital & HER2-specific CAR T cell & $\begin{array}{l}\text { HER2-positiive recurrent } \\
\text { or Refractory CNS } \\
\text { tumors }\end{array}$ \\
\hline & NCT02208362 & City of hope medical center & IL13Ra2-specific CAR T cell & $\begin{array}{l}\text { Recurrent or Refractory } \\
\text { high grade glioma }\end{array}$ \\
\hline \multirow[t]{4}{*}{$\begin{array}{l}\text { Checkpoint } \\
\text { blockade }\end{array}$} & NCT02502708 & $\begin{array}{l}\text { Children's healthcare of } \\
\text { atlanta }\end{array}$ & $\begin{array}{l}\text { IDO pathway inhibitor } \\
\text { plus indoximod and } \\
\text { Temozolomide }\end{array}$ & $\begin{array}{l}\text { Progressive malignant } \\
\text { brain tumors (including } \\
\text { DIPG) }\end{array}$ \\
\hline & NCT02359565 & $\begin{array}{l}\text { National cancer institute/ } \\
\text { Children's national medical } \\
\text { center (PBTC) }\end{array}$ & Pembrolizumab & $\begin{array}{l}\text { Recurrent, refractory } \\
\text { high grade glioma, } \\
\text { DIPG, Hypermutated } \\
\text { tumors, Ependymoma, } \\
\text { Medulloblastoma }\end{array}$ \\
\hline & NCT02992964 & $\begin{array}{l}\text { The hospital for Sick } \\
\text { children }\end{array}$ & Nivolumab & Hypermutant tumors \\
\hline & NCT03130959 & Bristol meyers squibb & Nivolumab, Ipilimumab & $\begin{array}{l}\text { High grade CNS } \\
\text { malignancies }\end{array}$ \\
\hline \multirow[t]{2}{*}{ Viral gene therapy } & NCT00634231 & Dana farber cancer institute & $\begin{array}{l}\text { ADV-tk + Prodrug therapy in } \\
\text { combination with radiation }\end{array}$ & $\begin{array}{l}\text { Malignant glioma } \\
\text { (WHO grade III or IV); } \\
\text { Recurrent ependymoma }\end{array}$ \\
\hline & NCT03330197 & Ziopharm & Ad-RTS-hIL-12 + Veledimex & $\begin{array}{l}\text { DIPG and Recurrent } \\
\text { malignant brain tumors }\end{array}$ \\
\hline Viral targets & NCT03299309 & Duke university & $\begin{array}{l}\text { PEP-CMV vaccine (human } \\
\text { pp65 and CMV glyocoprotein } \\
B+K L H)\end{array}$ & $\begin{array}{l}\text { Recurrent } \\
\text { medulloblastoma and } \\
\text { Malignant glioma }\end{array}$ \\
\hline
\end{tabular}




\begin{tabular}{|l|l|l|l|l|}
\hline Oncolytic virus & NCT02962167 & $\begin{array}{l}\text { University of california, San } \\
\text { francisco (PNOC) }\end{array}$ & $\begin{array}{l}\text { Modified measles vaccine } \\
\text { (MV-NIS) }\end{array}$ & $\begin{array}{l}\text { Recurrent } \\
\text { medulloblastoma or } \\
\text { ATRT }\end{array}$ \\
\hline NCT03178032 & $\begin{array}{l}\text { Clinica universidad de } \\
\text { navarra }\end{array}$ & $\begin{array}{l}\text { DNX-2401 oncolytic virus } \\
\text { targeting abnormal RB } \\
\text { pathway }\end{array}$ & DIPG \\
\hline
\end{tabular}

pediatric neuro-oncology consortium (PNOC) has an open phase I study for a H3.3K27M peptide vaccine for HLA-A2+ children with diffuse intrinsic pontine glioma and high grade glioma (NCT02960230). Diffuse intrinsic pontine glioma (DIPG) is an expansile glial tumor of the pons most commonly seen in children 5 to 9 -years-old [16]. Although this tumor only occurs in around $200 \mathrm{ca}$ ses per year, it is the focus of many clinical trials given its dismal prognosis of $<1 \% 5$ year overall survival and median overall survival of 11 months. Histone 3.3 and 3.1 mutations are present in over $80 \%$ of DIPG and $30 \%$ of glioblastoma [17]. The most common mutation causes a lysine to methionine switch at position 27 in one allele of H3F3A. This mutation re-programs the epigenetic landscape resulting in alterations in gene expression which then drive tumorigenesis. Epigenetic regulation of gene expression is a notoriously difficult target for treatment and immunotherapy is one avenue researchers are pursuing to address this.

Other vaccine trials in pediatric neuro-oncology include a glioma-associated antigen vaccine in HLA-A2+ children with DIPG or recurrent low or high grade tumors (NCT01130077) and a tumor lysate pulsed dendritic cell vaccine for high grade glioma (NCT01808820). A heat shock protein vaccine (HSPPC-96) (NCT02722512) and synthetic long peptide personalized neo-epitope vaccine (NCT03068832) are also being conducted in pediatrics.

In the area of adoptive cellular immunotherapy, a chimeric antigen receptor (CAR) T cell with specificity to HER2 is available in a phase I clinical trial for recurrent HER2-positive recurrent or refractory pediatric CNS tumors (NCT03500991). This trial is evaluating the safety and efficacy of autologous CD4 and CD8 T cells transduced with a lentivirus to express a HER2-specific CAR. The CAR T cell product is then administered directly into the tumor resection cavity or ventricular system using an indwelling catheter. Patients can receive anywhere from two to six courses of CAR T cell infusion depending on how they tolerate the therapy. In another open trial, an IL13R $\alpha 2$-specfic, 41BB costimulatory, CAR T cell product is administered weekly for three weeks into an intratumoral or intraventricular catheter (NCT02208362). Preliminary evidence of antitumor activity of an anti-disialoganglioside (GD2) CAR T cell in H3.3K27M midline glioma orthotopic xenograft models will also likely lead to a new CAR T cell trial for DIPG and H3.3K27M mutant tumors [18]. Xenograft models for these midline tumors revealed significant inflammation at the site of the tumor which did result in lethal hydrocephalus in a few of the animals. Investigators suggest neurointensive care management will be essential in the upcoming in-human trial [15].

Checkpoint inhibition has also been an active area of research for pediatric brain tumors. The Pediatric brain tumor consortium (PBTC) has developed a phase I trial evaluating the safety and feasibility pembrolizumab for recurrent or progressive DIPGs, high grade gliomas, hypermutant tumors, ependymoma, and medulloblastoma (NCT02359565). Early data from this trial revealed challenges in management of peritumoral inflammation and pseudoprogression for tumors located in the brainstem and posterior fossa, leading to subsequent trial design alterations in order to maximize the safety of this approach. The results of CheckMate-143 for adult glioblastoma revealed tolerability and early efficacy data of the combination of nivolumab and ipilumumab (PD1 and CTLA4 inhibition, respectively) $[19,20]$. This trial is also now open to pediatric high grade CNS malignancies on CheckMate-908 (NCT03130959).

Viral based gene therapies have also expanded into pediatric neuro-oncology. Therapeutic IL-12 has been under investigation because of IL-12's ability to bind IL12 receptors on natural killer (NK) cells and T cells and its ability to polarize $T$ cell differentiation towards type 1 helper T cells (Th1) and interference with regulatory T cell differentiation [21]. Unfortunately, it is difficult to use therapeutically because it is unstable by itself and has a short half-life. Ad-RTS-hIL-12 is a viral product that uses a replication incompetent adenoviral vector delivery of the IL-12 gene under regulation of an activator [22]. The IL-12 expression is then regulated by an oral activator ligand known as INXN-1001 or veledimex. This product is administered intratumorally and allows inducible gene expression through administration of a small molecule ligand. In adults, the gene therapy was found to be tolerable with a reasonable safety profile [23]. This trial is also open for pediatric patients with DIPG or recurrent malignant brain tumors (NCT03330197). Similarly, a trial using an adenoviral vector containing the herpes simplex virus thymidine kinase gene (ADVtk) plus prodrug, valacyclovir, is being tried in combination with radiation and temozolomide in pediatric brain tumors (NCT00634231). This therapy works by the phosphorylation of the valacyclovir by the HSV-tk converting the drug into nucleotide analogs which are toxic to dividing or actively repairing cells [22]. This effect is thought to be potentiated by the concurrent use of DNA-damaging therapy, such as radiation and chemotherapy and has been safely delivered in adults [24]. 
In the area of oncolytic viruses, PNOC has opened a phase I study of an attenuated modified measles virus (MV-NIS) for the treatment of recurrent medulloblastoma and atypical teratoid rhabdoid tumor (ATRT) (NCT02962167). This oncolytic virus contains a human thyroidal sodium iodide symporter (NIS) that allows evaluation of the distribution and cytotoxic effect of the measles virus on SPECT imaging [25]. The MV-NIS is injected into the tumor bed or subarachnoid space. Oncolytic viruses work by taking advantage of selective viral replication and killing in cancer cells that do not possess the cellular repair mechanisms of normal cells [26]. This selective killing also induces a systemic anti-tumor immune response. A survival benefit has been observed with the oncolytic virus polio/rhinovirus recombinant (PVSRIPO) in treatment of adult glioblastoma and is being accelerated for FDA approval [27]. Oncolytic poliovirus is administered intracerebrally by convection-enhanced delivery (CED) and is now in an open phase $1 \mathrm{~b}$ trial for children with recurrent WHO III or IV malignant glioma (NCT03043391). Immunotherapy trials in pediatric neuro-oncology are not limited to the United States. In Spain, a Phase I trial for DNX2401, an oncolytic virus targeting tumor cells with abnormalities in the RB pathway, is open for patients with DIPG (NCT03178032). The virus will be injected through a catheter using the same trajectory as the initial biopsy and the virus will infect cells through integrins which are abundant in gliomas. If DNX-2401 is safe in children, this trial will be moved to a multi-institutional trial in Europe.

As many of these new and exciting therapies remain in Phase I or I/II studies without published data, it is difficult to assess what immunotherapy strategies are the most advantageous or how they compare against each other in terms of efficacy. Based on previous research, monotherapy with checkpoint blockade appears to be most effective against tumors with high mutational burden [28-30]. This suggests pediatric brain tumors may require combination immunotherapy that also ignites antigen presentation and recognition, such as vaccine therapy in combination with checkpoint blockade. In a previously reported phase I trial for glioma-associated antigen (GAA) epitope peptide vaccines in HLA-A2 positive children with newly diagnosed brainstem glioma and high grade glioma, all children had positive enzyme-linked immunosorbent spot analysis for anti-GAA immune response [31]. Of 26 children enrolled, $19 \mathrm{pa}-$ tients had stable disease, 2 had partial responses, 1 had a minor response, and 2 had prolonged disease-free status after surgical resection. In a pilot study evaluating tumor lysate pulsed dendritic cell vaccine in 3 pediatric patients with high grade glioma, moderate cytokine profile changes were observed in peripheral blood of patients and, in one patient with recurrent disease post vaccination, limited immune infiltrate was seen in resected tissue [32]. Another effective strategy may be immunotherapies that utilize antigen exposure during radiation. During radiation therapy, tumor antigens are exposed and processed by antigen presenting cells for presentation $[33,34]$. If immunotherapy treatment aimed at expanding the immune response can be given in close proximity to the radiation therapy, it may result in more robust immune-mediated cytolytic activity. Also, CAR T cells have been promising in preclinical models and also bypass the need for antigen presentation and $T$ cell activation [18]. In tumors with low mutational burden, this may be an effective strategy. In a patient-derived diffuse midline glioma orthotopic xenograft model, a GD2-targeted CAR T cell resulted in complete responses in all five models with only small numbers of residual H3.3K27M+ tumor cells remaining [18]. This treatment will soon enter a phase I clinical trial.

Overall, immunotherapy-based clinical trials for pediatric brain tumors span multiple modalities including checkpoint inhibition, vaccine therapy, adoptive cellular immunotherapy, and virally-mediated immunotherapies. This wide breadth of trial activity highlights the need for better treatments for pediatric malignant brain tumors and emphasizes the excitement around immunotherapy for pediatric patients who face a multitude of long term sequelae of current radiotherapy and chemotherapy regimens.

\section{Challenges Facing Immunotherapy for Pediatric Neuro-Oncology}

The challenge of pseudoprogression is not unique to pediatric neuro-oncology. Experience from immunotherapy trials for adult brain tumors revealed a need for specific disease assessment criteria unique to this field of research. As clinicians and radiologists attempted to distinguish tumor related inflammation from tumor growth on MRI's for patients on immunotherapy trials, they realized the standard response assessment criteria in neuro-oncology (RANO) was insufficient. Patients who are experiencing a positive immune response from treatment and subsequent inflammation at the tumor site would be typically labeled 'progressive disease' based on strict RANO criteria. A new schematic was developed know as the immunotherapy response assessment criteria for neuro-oncology (iRANO) [35]. It incorporates repeated MRI scans for patients where pseudoprogression versus true progression is unclear and they are within the first 6 months of initiation of immunotherapy. It also adds the use of particular MRI images like T2 FLAIR to try to tease out this issue of pseudoprogression as well.

The iRANO criteria are useful in evaluation of supratentorial lesions and in the context of minimal clinical decline observed in the setting of pseudoprogression. However, in pediatrics most of the tumors being evaluated are in very sensitive areas of the brain, namely the posterior fossa, so significant symptomatology related to pseudoprogression is not uncommon and can require 
early initiation of steroids or discontinuation of therapy. Swelling of a posterior fossa tumor can result in hydrocephalus via compression of the $4^{\text {th }}$ ventricle causing the patient to experience headache, nausea, and vomiting. Peritumoral swelling of brainstem tumors can result in motor dysfunction and cranial nerve deficits impairing swallowing and even leading to apnea and death. In the open PBTC trial for pembrolizumab, initial signs of progression resulted in temporary closure in order to tease out the impact of possible pseuodoprogression in this patient population. Due to the unique mechanism of action for immunotherapy, traditional measures of progression, including MRI 'growth' or appearance of new obvious disease sites with or without subsequent clinical deterioration, may not be reliable. Novel imaging modalities such as ferumoxytol imaging, novel PET isotopes, and spectroscopy are being investigated in order to more clearly delineate this difference.

Steroid use for pediatric brain tumors is very common and is exacerbated by treatment with radiation. The goal of most immunotherapy trials is to limit or decrease dosing to a physiologic steroid level. Some clinical trials have required less than $0.5 \mathrm{mg} / \mathrm{kg} / \mathrm{day}$ of steroids, although even that restriction likely impairs the efficacy of immunotherapy [36]. For example, patients with diffuse intrinsic pontine glioma (DIPG) often require initiation of steroids at diagnosis for cranial nerve deficits and most receive dexamethasone during radiation treatment. It is sometimes impossible to wean steroid doses due to significant symptoms, which may be worsened by initiation of immunotherapy. This presents unique ethical considerations when testing immunotherapy. For instance, should invasive supportive measures, such as, NG tubes or mechanical ventilation be undertaken for patients receiving phase I experimental agents? When can a diagnosis of pseudoprogression be made, and how can parents be advised when making decisions surrounding heroic measures, such as intubation or resuscitation? How do we view these same decisions in patients with DIPG who possess $<1 \%$ chance of survival at 5 years?

The location of the majority of pediatric brain tumors makes the use of immunotherapy a challenge both in terms of pseudoprogression and the use of steroids for symptom management. For those designing clinical trials in immunotherapy for pediatric brain tumors, there is a tight balance between patient safety and accurate evaluation of efficacy of these therapies.

\section{Conclusions}

Immunotherapy for pediatric malignancies has had recent successes in acute leukemia and neuroblastoma. However, future immunotherapy research in pediatric oncology will need to address the challenges of low mutational load tumors and potentially limited immunogenicity, differences in pediatric and adult immune infiltrate, and nuances to management of toxicity of im- munotherapy in children. The applications in pediatric neuro-oncology continue to present additional obstacles involving appropriate clinical trial design and management of pseudoprogression and steroid use, although the refinement of IRANO and accumulation of experience with immunotherapy in the central nervous system hold hope for the future.

\section{References}

1. Ozkaynak MF, Gilman AL, London WB, Narajo A, Dicclanni $\mathrm{MB}$, et al. (2018) A comprehensive safety trial of chimeric antibody 14.18 with GM-CSF, IL-2, and isoretinoin in high risk neuroblastoma patients following myeloablative therapy: Children's Oncology Group study ANBL0931. Front Immunol 9: 1355.

2. Castel V, Segura V, Canete A (2010) Treatment of high risk neuroblastoma with anti-GD2 antibodies. Clin Transl Oncol 12: 786-793.

3. $\mathrm{Yu} A \mathrm{~L}$, Gilman AL, Ozkaynak MF, London WB, Kreisman SG, et al. (2010) Anti-GD2 antibody with GM-CSF, interleukin-2, and isotretinoin for neuroblastoma. $\mathrm{N}$ Engl $\mathrm{J}$ Med 363: 1324-1334.

4. McKerrow KJ, Mackie RM, Lesko MJ, Pearson C (1988) The effect of oral retinoid therapy on the normal human immune system. British J Derm 119: 313-320.

5. Dispenza MC, Wolpert EB, Gilliland KL, Dai P, Cong Z, et al. (2012) Systemic isotretinoin therapy normalizes exaggerated TLR-2-mediated innate immune responses in acne patients. J Invest Dermatol 132: 2198-2205.

6. Minard-Colin V, Auperin A, Pillon M, Burke A, Anderson JR, et al. (2016) Results of the randomized intergroup trial interB-NHL Ritux 2010 for children and adolescents with high risk B-cell non-Hodgkin's lymphoma and mature acute leukemia: Evaluation of Rituximab efficacy in addition to standard LMB chemotherapy regimen. J Clin Oncol 34: 10507.

7. Buechner J, Grupp SA, Maude SL, Bover M, Bittencourt H, et al. (2017) Global registration trial of efficacy and safety of CTL019 in pediatric and young adult patients with relapsed/refractory acute lymphoblastic leukemia (ALL): Update to the interim analysis. European Hematologic Association.

8. Vogelstein B, Papadopoulos N, Velculescu V, Zhou S, Diaz LA, et al. (2013) Cancer genome landscapes. Science 339: 1546-1558.

9. Simon KA, Hollander GA, McMichael A (2015) Evolution of the immune system from infancy to old age. Proc Biol Sci 282: 2043065.

10. Yang I, Han SJ, Sughrue ME, Tihan T, Parsa AT (2011) Immune cell infiltrate differences in pilocytic astrocytoma and glioblastoma: evidence of distinct immunological microenvironments that reflect tumor biology. J Neurosurg 115: 505-511.

11. Yang I, Tihan T, Han SJ, Wrensch MR, Wiencke J, et al. (2010) CD8+ T cell infiltrate in newly diagnosed glioblastoma is associated with long-term survival. $\mathrm{J}$ Clin Neurosci 17: $1381-1385$.

12. Dunn GP, Fecci PE, Curry WT (2012) Cancer immunoediting in malignant glioma. Neurosurgery 71: 201-222.

13. Griesinger AM, Birks DK, Donson AM, Amani V, Hoffman LM, et al. (2013) Characterization of distinct immunophenotypes across pediatric brain tumor types. J Immunol 191: 1301966.

14. Griesinger AM, Donson AM, Foreman NF (2014) Immunotherapeutic implications of pediatric brain tumors. Oncoimmunology 3: e27256. 
15. Plant AS, Koyama S, Sinai C, Solomon IH, Griffin GK, et al. (2018) Immunophenotyping of pediatric brain tumors: correlating immune infiltrate with histology, mutational load, and survival and assessing clonal $\mathrm{T}$ cell response. J Neurooncol 137: 269-278.

16. Sophie FM, Van Zanten V, Baugh J, Chaney B, De Jongh D, et al. (2017) Development of the SIOPE DIPG network, registry, and imaging repository: A collaborative effort to optimize research into a rare and lethal disease. J Neurooncol 132: 255-266.

17. Chan KM, Fang D, Gan H, Hashizume R, Schroeder M, et al. (2013) The histone H3.3K27M mutation in pediatric glioma reprograms $\mathrm{H} 3 \mathrm{~K} 27 \mathrm{M}$ methylation and gene expression. Genes Dev 27: 965-990.

18. Mount CW, Majzner RG, Sundaresh S, Arnold EP, Kadapakkam M, et al. (2018) Potent antitumor efficacy of anti-GD2 CAR T cells in H3-K27M+ diffuse midline gliomas. Nat Med 24: 572-579.

19. Sampson JH, Vlahovic G, Sahebjam S, Omuro AM, Baehring JM, et al. (2015) Preliminary safety and activity of nivolumab and its combination with ipilimumab in recurrent glioblastoma (GMB): CHECKMATE-143. J Clin Oncol 15: 3010-3010.

20. Omuro M, Vlahovic G, Lim M, Saheblam S, Baehring J, et al. (2018) Nivolumab with or without ipilimumab in patients with recurrent glioblastoma: results from exploratory phase I cohorts of CheckMate 143. Neuro Oncol 20: 674-656.

21. Tugues S, Burkhard SH, Ohs J, Vrohlings M, Nussbaum K, et al. (2015) New insights into IL-12 mediated tumor suppression. Cell Death Differ 22: 237-246.

22. Barrett JA, Cai H, Miao J, Khare PD, Gonzalez P, et al. (2018) Regulated intratumoral expression of IL-12 using a RheoSwitch Therapeutic System (RTS) gene switch as gene therapy for treatment of glioma. Cancer Gene Ther 25: 106-116.

23. Lebel FM, Barrett JA, Chiocca EA, Yu J, Lukas RV, et al. (2016) Effect of controlled intratumoral viral delivery of AdRTS-hIL-12 + oral veledimex in subjects with recurrent or progressive glioma. J Clin Oncol 15: 2052-2052.

24. Chiocca EA, Aguilar LK, Bell SD, Kaur B, Hardcastle J, et al. (2011) Phase IB study of gene-mediated cytotoxic immunotherapy adjuvant to up-front surgery and intensive timing radiation for malignant glioma. J Clin Oncol 29: 3611-3619.

25. Hutzen B, Pierson CR, Russell CJ, Galanis E, Raffel C et al. (2012) Treatment of medulloblastoma using an oncolytic measles virus encoding the thyroidal sodium iodide symporter shows enhanced efficacy with radioiodine. BMC Cancer 12: 508 .
26. Aurelial L (2016) Oncolytic viruses as immunotherapy: progress and remaining challenges. OncoTargets 9: 26272637.

27. Desjardins A, Sampson JH, Peters KB, Vlahovic G, Randazzo S, et al. (2016) Patient survival on the dose escalation phase of oncolytic Polio/Rhinovirus recombinant (PVSRIPO) against WHO grade IV malignant glioma (MG) clinical trial compared to historical controls. J Clin Oncol 34: 2061

28. Duraiswany J, Kaluza KM, Freeman GJ, Coukos G (2013) Dual blockade of PD-1 and CTLA-4 combined with tumor vaccine effectively restores $T$ cell rejection function in tumors. Cancer Res 73: 3591-3603.

29. Borghaei H, Paz-Ares L, Horn L, Spigel DR, Steins M, et al. (2015) Nivolumab versus docetaxel in advanced nonsquamous non-small-cell lung cancer. NEJM 373: 1627-1639.

30. Snyder A, Makarov V, Merghoub T, Yuan J, Zaretsky JM, et al. (2014) Genetic basis for clinical response to CTLA-4 blockade in melanoma. NEJM 37: 2189-2199.

31. Pollack IF, Jakacki RI, Butterfield LH, Hamilton RL, Panigraphy A, et al. (2014) Antigen-specific immune responses and clinical outcome after vaccination with glioma-associated antigen peptides and polyinosinic-polycytidlylic acid stabilized by lysine and carboxymethylcellulose in children with newly diagnosed malignant brainstem gliomas. J Clin Oncol 32: 2050-2058.

32. Lasky JL, Panosyan EH, Plant A, Davidson T, Yong WH, et al. (2013) Autologous tumor lysate-pulsed dendritic cell immunotherapy for pediatric patients with newly diagnosed or recurrent high-grade gliomas. Anticancer Res 33: 20472056.

33. Mohamed O, Diaz de Leon A, Schroeder S, Leiker A, Christie A, et al. (2018) Safety and efficacy of concurrent immune checkpoint inhibitors and hypofractionated body radiotherapy. Oncoimmunology 7: e1440168.

34. Vandenabeele P, Vandecasteele K, Bachert C, Krysko O, Krysko DV (2016) Immunogenic apoptotic cell death and anticancer immunity. Adv Exp Med Biol 930: 133-149.

35. Okada H, Weller M, Huang R, Finocchiaro G, Gilbert MR, et al. (2015) Immunotherapy response assessment in neuro-oncology: A report of the RANO working group. Lancet Oncol 16: e534-e542.

36. Margolin K, Ernstoff M, Hamid O, Lawrence D, McDermott D, et al. (2012) Ipilimumab in patients with melanoma and brain metastases: An open-label, phase 2 trial. Lancet Oncol 13: 459-465. 